\title{
Mobility oscillations of electrons on thin He films
}

\author{
D. Cieslikowski and P. Leiderer \\ Fachbereich Physik, Johannes Gutenberg-Unitersitïl, Mainz, Federal Republic of Germany \\ AND \\ A. J. DAHM \\ Case Western Reserve University. Cleveland, OH 44106, U.S.A.
}

Received Octobcr 13,1986

\begin{abstract}
We have studied electrons above liquid films of ${ }^{3} \mathrm{He}$ and ${ }^{4} \mathrm{He}$ condensed on various solid substrates. For helium films on solid hydrogen. pronounced oscillations of the clectron mobility are observed as the film thickness is changed. The oscillations are related to the formation of layers of the liquid close to the wall. Our results indicate substantial layering of the films up to nearly 10 atomic distances.
\end{abstract}

\begin{abstract}
Nous avons étudié les électrons au-dessus de films liquides de ${ }^{3} \mathrm{He}$ et ${ }^{4} \mathrm{He}$ condensés sur différents substrats solides. Pour des films d'hélium sur hydrogène solide. on observe des oscillations prononcées de la mobilité des électrons lorsque l'épaisseur du film est changée. Ces oscillations sont reliées à la formation de couche du liquide près de la paroi. Nos résultats indiquent que la formation de couches dans les films est importante jusqu'à presque 10 distances atomiques.
\end{abstract}

Can. J. Phys, 65, $1525(1987)$

\section{Introduction}

Among the various probes for studying surfaces, free clectrons probably play the most important role nowadays. More and more sophisticated techniques have been developed to investigate the structure and the excitation of surfaces by low energy electron diffraction, electron energy loss spectroscopy, etc. These methods usually require ultra high vacuum conditions and are therefore suitable only for studying solid surfaces. For liquids the vapor pressure in general is so high and hence the mean free path of the electrons so short that these techniques are not applicable. Illere we discuss a method that although quite different from the conventional electron spectroscopies, also makes use of free (or rather quasi-free) electrons to obtain information about substrate surfaces via the interaction of the electrons with these substrates. This method relies on materials with a negative electron affinity and is therefore, in particular, suited for the quantum liquids and solids formed by helium hydrogen; it can be applied even under conditions where the mean free path of the electrons is relatively short.

In the work presented here we have used the electrons to study thin liquid films of He covering solid substrates, mainly solid $\mathrm{H}_{2}$. Before describing our results, we give a brief outline of the behavior of electrons on bulk liquid He. Some reviews on this subject are listed in ref. 1.

\section{Electrons on bulk liquid He}

Electrons on the surface of liquid $\mathrm{He}$ are trapped in a potential well due to the image field that confines the electrons to movement parallel to the surface. A potential barrier of $10^{4} \mathrm{~K}$ prevents the electrons from entering the liquid. At low temperatures $(T \leqq 1 \mathrm{~K}$ ) only the ground state (bound with an energy of $10 \mathrm{~K}$ ) is populated, where the electrons have a distance of about $100 \AA$ from the liquid. The interaction with the surface is thus relatively weak, giving rise to a high mobility $\mu$ of these "surface electrons."

Two mechanisms govern the electron mobility in this case. At $T<0.7 \mathrm{~K}, \mu$ is essentially determined by scattering of the electrons from ripplons on the superfluid surface, leading to only a weak temperature dependence, as shown in Fig. 1. For higher $T$ the increasing number of atoms in the gas phase

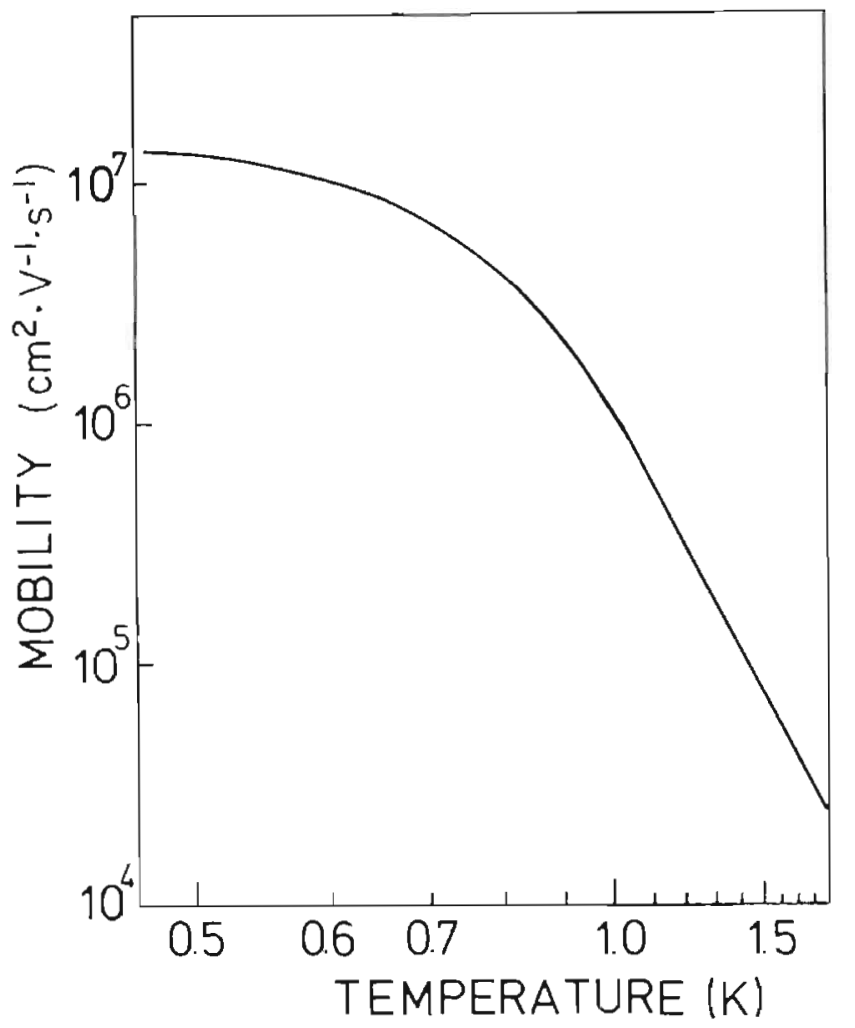

FIG. 1. Electron mobility on bulk ${ }^{4} \mathrm{He}$ (from ref. 1).

dominates the scattering, and as a result the mobility drops rapidly. In addition to these "single clectron" effects, a strong influence on the mobility is observed when the 2-dimensional electron layer undergoes, at low enough $T$, a phase transition to an electron solid, the so-called Wigner crystal $(1,2)$.

\section{Electron density on He films}

In the context of Wigner crystallization, interest in electrons on films arose because one expects to reach higher electron densities, and thus wider regions in the phase diagram, on a film as compared to the bulk liquid. In the latter case a fundamental limit is set by the electrohydrodynamic (EHD) 
instability of the charged surface, which develops for electron densities above a critical value $n_{c}$. The EHD instability is a result of the softening of ripplons in the presence of the electron layer, described by the dispersion relation for a charged surface (3):

[1] $\omega^{2}=g k+\frac{\sigma}{\rho} k^{3}-\frac{4 \pi n^{2} e^{2}}{\rho} k^{2}$

Here $\omega$ and $k$ are the ripplon frequency and wave vector, respectively, $g$ is the acceleration due to gravity, $\sigma$ the surface tension, $\rho$ the density of the liquid, and $e$ the electronic charge. According to [1], $\omega$ becomes imaginary and the surface therefore unstable at

[2] $n_{\mathrm{c}}=\frac{1}{e}\left(\rho g \sigma / 4 \pi^{2}\right)^{1 / 4}$

which for ${ }^{4} \mathrm{He}$ at $1 \mathrm{~K}$ yields a value of $n_{\mathrm{c}}=2.4 \times 10^{9} \mathrm{~cm}^{-2}$.

For films, [1] is modified due to the van der Waals interaction of the He with the substrate:

$$
\text { [3] } \begin{aligned}
\omega^{2}=\frac{\rho_{\mathrm{s}}}{\rho}\left[\left(\frac{3 \alpha}{\rho d^{4}}+g\right) k\right. & +\frac{\sigma}{\rho} k^{3} \\
& \left.-\frac{4 \pi n^{2} e^{2}}{\rho} k^{2} F(k, \varepsilon)\right] \tanh (k d)
\end{aligned}
$$

The factor $\rho_{s} / \rho$ takes into account that only the superfluid fraction of the liquid can move in the film, $\tanh (k d)$ arises from the finite thickness $d$ of the liquid, and the factor $F$ is approximately equal to the dielectric constant $\varepsilon$ of the solid substrate. The van der Waals contribution $3 \alpha / \rho d^{4}$ in effect increases the term linear in $k$ by many orders of magnitude over the pure gravitational contribution. The critical charge density should therefore be considerably increased compared to [2]. Indeed, electrons on films have been observed to be stable up to $10^{11} \mathrm{~cm}^{-2}$. This was found for a wide class of substrates, insulating as well as metallic, and irrespective of the film thickness before charging. The latter result, somewhat surprising in view of [3], could be explained on the basis of ellipsometric measurements of the thickness of charged films (see Fig. 2). Due to the electrostatic pressure of the electron layer the thickness is reduced (4) to

[4] $d=\left(1 / d_{0}^{3}+2 \pi n^{2} e^{2} / \alpha\right)^{-1 / 3}$

where $d_{0}$ is the thickness of the film before charging (given, e.g., by $d_{0}=(\alpha / \rho g h)^{1 / 3}$ for a film at a height $h$ above the bulk liquid level).

In an additional measurement we investigated the influence of the surface electrons on the propagation of third sound in the He films. A pronounced increase of the third sound velocity was observed on charging, which could be completely attributed, however, to the reduction of film thickness by the charges according to [4]. An indication of a decrease of the velocity, which could have been taken as a precursor of the EHD instability in the film, was not found even for the maximum attainable charge density of $10^{11} \mathrm{~cm}^{-2}$, where the (average) film thickness is less than $100 \AA$. We therefore believe that the process that limits the maximum electron density on He films is not a collective EHD instability, but rather a single particle process like the tunneling of individual electrons, probably through "weak spots" of the film at imperfections of the substrate.

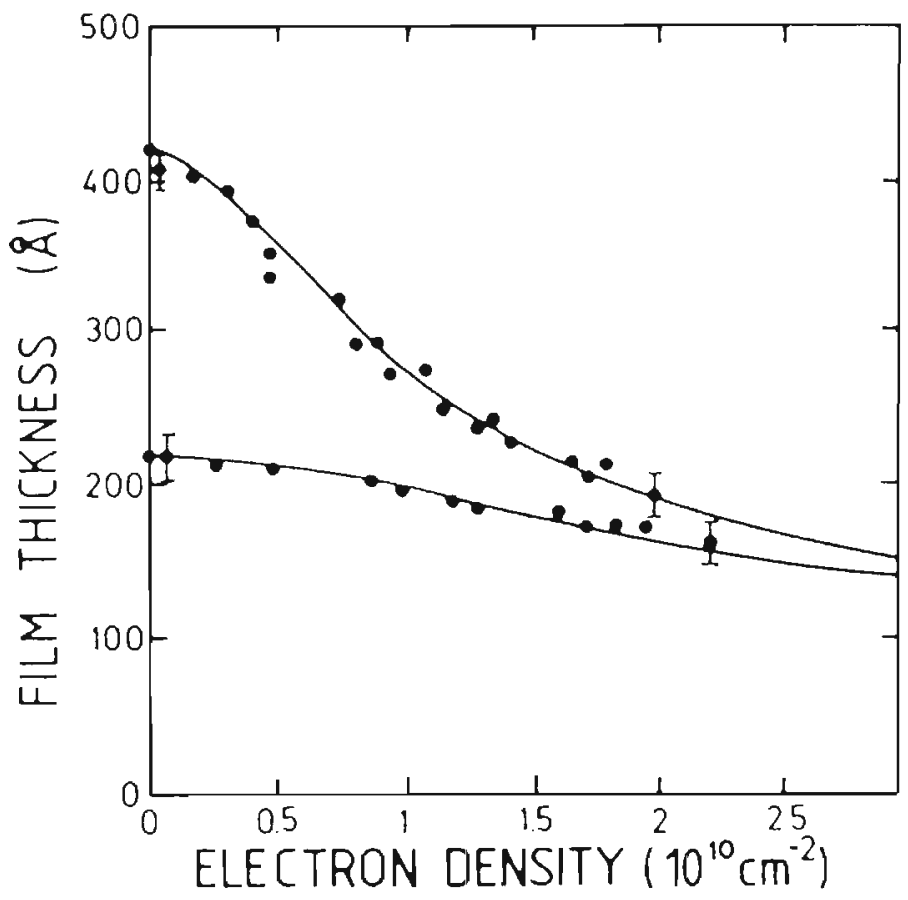

FIG. 2. Thickness $d$ of two charged ${ }^{4} \mathrm{He}$ films as a function of the electron density. The thickness $d_{0}$ of the uncharged films was 220 and $420 \AA$, respectively (ref. 4 ).
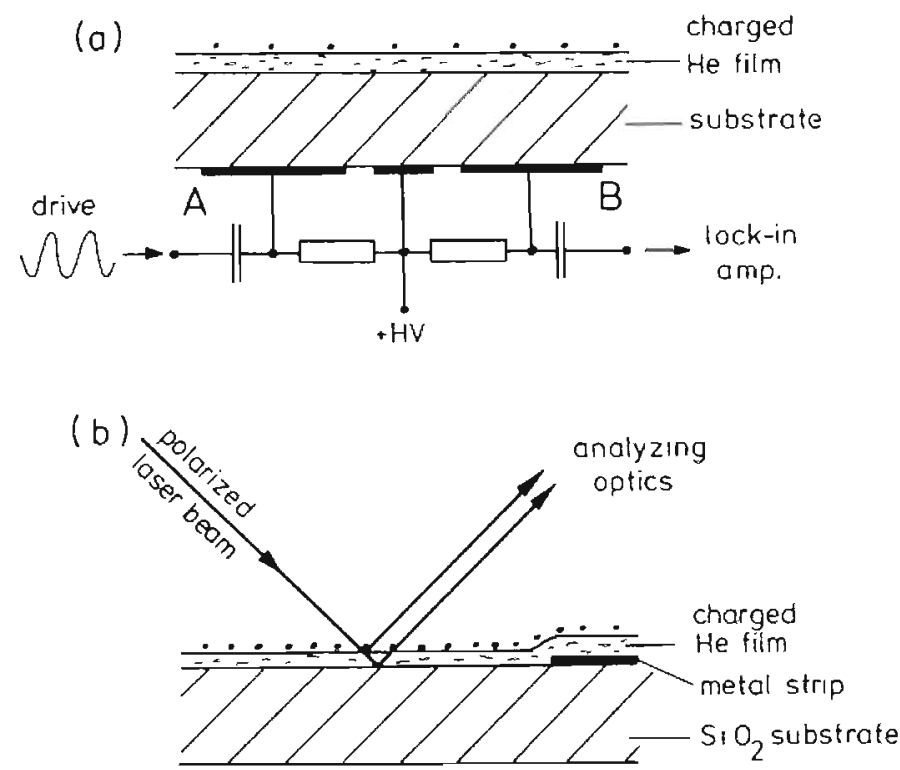

FIG. 3. Schematic experimental setups for measuring the mobility of electrons on He films. (a) Sommer technique. applied for mobilities $\mu>1 \mathrm{~cm}^{2} \mathrm{~V}^{-1} \mathrm{~s}^{-1}$; a typical value for the driving frequency is $100 \mathrm{kHz}$. (b) Ellipsometric tcchnique (ref. 4).

\section{Electron mobility on He films}

Measurements of the mobility of surface electrons on He have been carried out with several techniques, like cyclotron resonance (1) or the "Sommer technique," where an arrangement with segmented capacitor plates is used, as shown in Fig. $3 a$ (5). Application of a small ac voltage (on the order of millivolts) to plate A gives rise to a modulation of the electron density above plate $B$ and hence to an ac voltage at $B$. Direct 


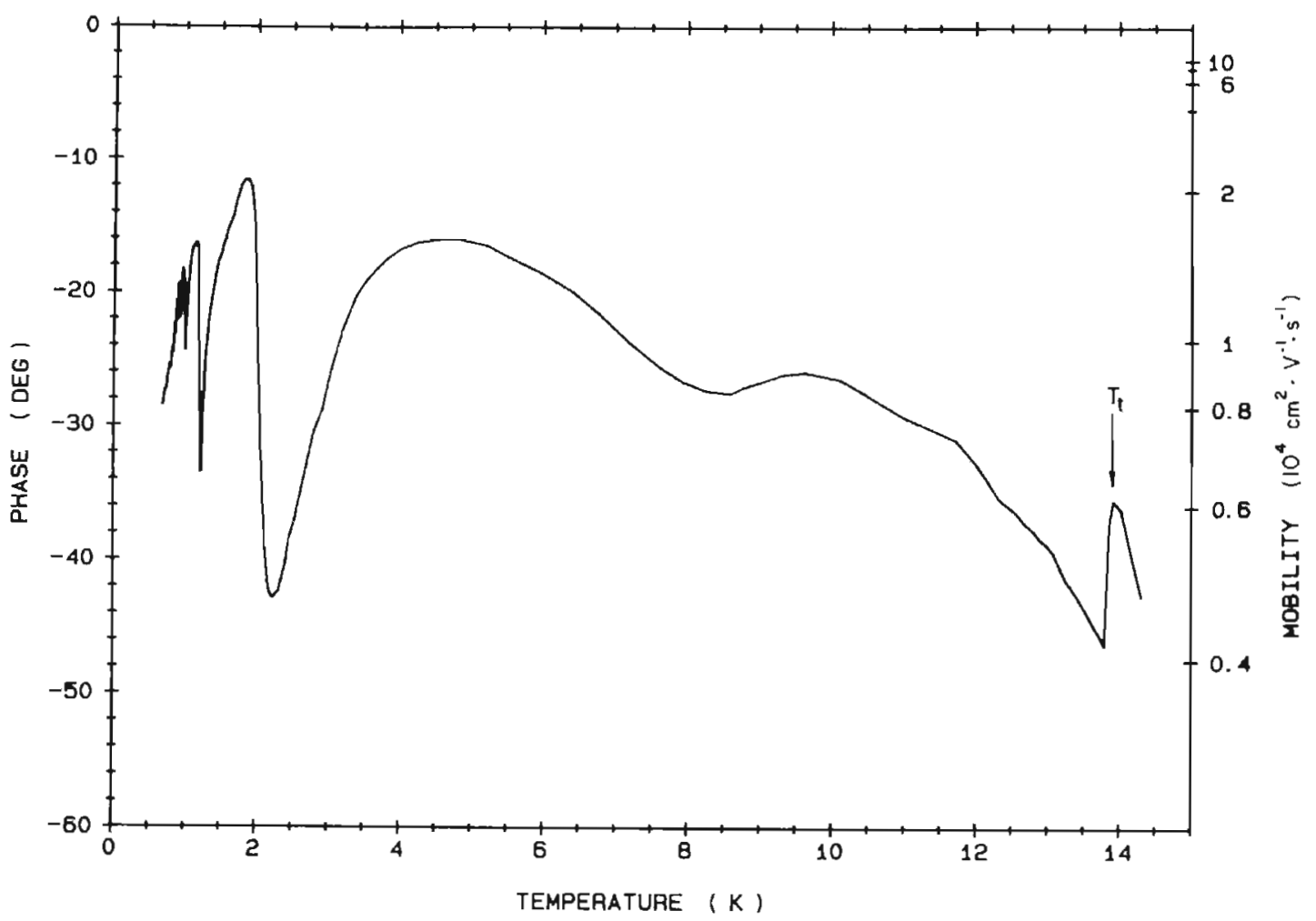

FIG. 4. Phase shift $\varphi$ and mobility $\mu$ of electrons on solid $\mathrm{H}_{2}$ covered by a He film with a temperature dependent thickness. For $T>5 \mathrm{~K}$ the data are equivalent to those on a bare $\mathrm{H}_{2}$ substrate. because essentially all the He is in the gas phase (pressure $p=0.35 \mathrm{mbar}$ at $5 \mathrm{~K}$ ). At the lowest temperatures, where all the He has condensed, the He film reaches a thickness of 10 layers. The electron density in this run was $5 \times$ $10^{8} \mathrm{~cm}^{-2}$.

crosstalk from A to $B$ can be reduced by a separating platc, which is on ac ground. The amplitude and phase of the signal due to the electrons contain information about the density and the mobility of the electrons. In particular, the phase shift with respect to the driving voltage, $\varphi$, is related to the mobility by

[5] $\tan \varphi \propto \mu^{-1}$

We have tried to measure with this technique the electron mobility on He films covering various substrates. For only one of these substrates, solid $\mathrm{H}_{2}$, was $\mu$ large enough that a measurement was possible; these experiments will be discussed in the next section. For all the other, more conventional, substrates like glass or Mylar the mobility was below the limit that can be detected with this setup, $\mu-1 \mathrm{~cm}^{2} V^{-1} \mathrm{~s}^{-1}$.

To obtain a better estimate on these low mobilities on films, we used the ellipsometric technique with a setup sketched in Fig. $3 b$. The method consists in measuring the electron density on the film via the film thickness, using [4]. In the beginning the film is charged to a high density, and then a positive potential is applied to the metallic strip evaporated onto part of the $\mathrm{SiO}_{2}$ substrate. As a result the electrons are pulled towards the strip, and the charge density above the insulating part of the substrate drops with a characteristic time constant inversely proportional to the mobility. Since the method allows measurement of variations in the charge density on the time scale of hours, it can also detect extremely small mobilities. For He films charged to a density of $10^{11} \mathrm{~cm}^{-2}$ we find $\mu<10^{-5} \mathrm{~cm}^{2} V^{-1} s^{-1}$ at $T=1.5 \mathrm{~K}$, which implies that under these conditions the electrons are essentially localized.

One possible mechanism that leads to a low electron mobil- ity on "usual" substrates (i.e., dielectric constants $\varepsilon>2$ ) has been discussed by Jackson and Platzman (6) and Andrei (7). Since the electrons, under the influence of the image charge in the substrate, exert a considerable pressure upon the surface, one expects the formation of dimples in the film underneath each individual electron. The electrons in this so-called polaron state have a considerably increased effective mass and hence a reduced mobility.

A second source for a decrease of $\mu$, which should become important for nonideal substrate surfaces, is the pinning of the electrons to surface irregularities. This process should be efficient especially at high electron densities, where the $2 \mathrm{D}$ electron system becomes strongly correlated, so that the pinning of only a few electrons can lead to the complete immobilization of the whole electron layer. It should be mentioned that at a temperature of $1.5 \mathrm{~K}$ and a density of $10^{11} \mathrm{~cm}^{-2}$ a Coulomb system is well within the Wigner crystal phase, which for an imperfect substrate may be polycrystalline or even a "Wigner glass." It appears interesting to determine the influence of the image charges on the phase diagram of such a system, which should be modified, due to dipolar contributions, as compared to the nearly pure Coulomb interaction between electrons on bulk He.

\section{Electron mobility on "low $\varepsilon$ " substrates}

One way to reduce the interaction of the electrons with the substrate and thus increase the mobility is to use a solid substrate with a dielectric constant close to $\mathrm{l}$, such as solid $\mathrm{H}_{2}$ or $\mathrm{Ne}(8,9)$. Before discussing such surfaces, in our case $\mathrm{H}_{2}$, covered with a thin He film, we first consider the bare substrate. 

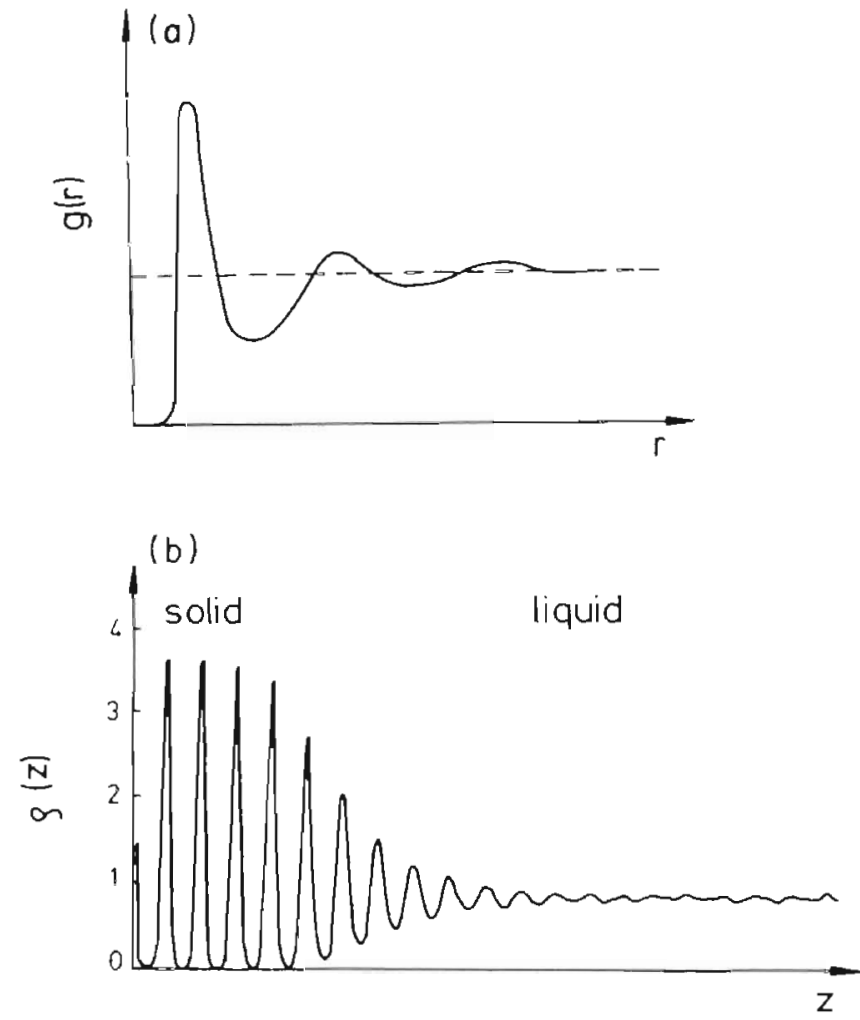

FIG. 5. (a) Radial distribution function of a simple classical fluid in 3D (schematic). (b) Density distribution in the vicinity of a van der Waals solid-liquid interface (from ref. 17).

Electrons on condensed hydrogen form a 2D layer similar to the system on bulk He, however, at a reduced distance from the surface $(z \sim 20 \AA)$ due to the larger polarizability of $\mathrm{H}_{2}$. The mobility of the electrons on the liquid phase (at temperatures above the triple point, $T_{\mathrm{t}}=13.8 \mathrm{~K}$ ) is dominated by gas atom scattering and therefore decreases rapidly as the temperature and hence the gas atom density increase. On the solid ( $T<$ $13.8 \mathrm{~K}$ ), additional scattering arises from surface defects, so that the inverse scattering time (proportional to the inverse mobility) is

$$
\text { [6] } \tau^{-1} \approx \tau_{\text {gas }}^{-1}+\tau_{\text {defect }}^{-1}
$$

An example for the electron mobility on $\mathrm{H}_{2}$ as a function of temperature is shown in Fig. 4. Since $\mu$ in this case is high we used here the technique sketched in Fig. $3 a$. The phase shift (related to the mobility by [5]) displays a sharp step at the triple point, indicating the additional scattering from surface defects on the solid. An absolute scale for $\mu$ was obtained from a measurement on the liquid substrate just above $T_{t}$, where several other experiments have yielded a value of $6 \times 10^{3} \mathrm{~cm}^{2} \mathrm{~V}^{-1} \mathrm{~s}^{-1}$ $(8,10)$.

On solid $\mathrm{H}_{2}$, the temperature dependence of $\mu$ should level off as $T$ is lowered, ${ }^{1}$ because the gas atom scattering becomes negligible, and the scattering from surface defects should not depend on $T$. Yet anomalies are observed in general for temperatures around $8 \mathrm{~K}$, in Fig. 4 only visible as a shallow dip, suggesting that some change on the surface takes place, such as, e.g., surface roughening or surface melting (refs. 8 and, for reviews, 11). A more trivial explanation, namely the devel-

\footnotetext{
${ }^{1}$ This only holds as long as the correlation effects in the electronic motion, already mentioned above, are negligible.
}

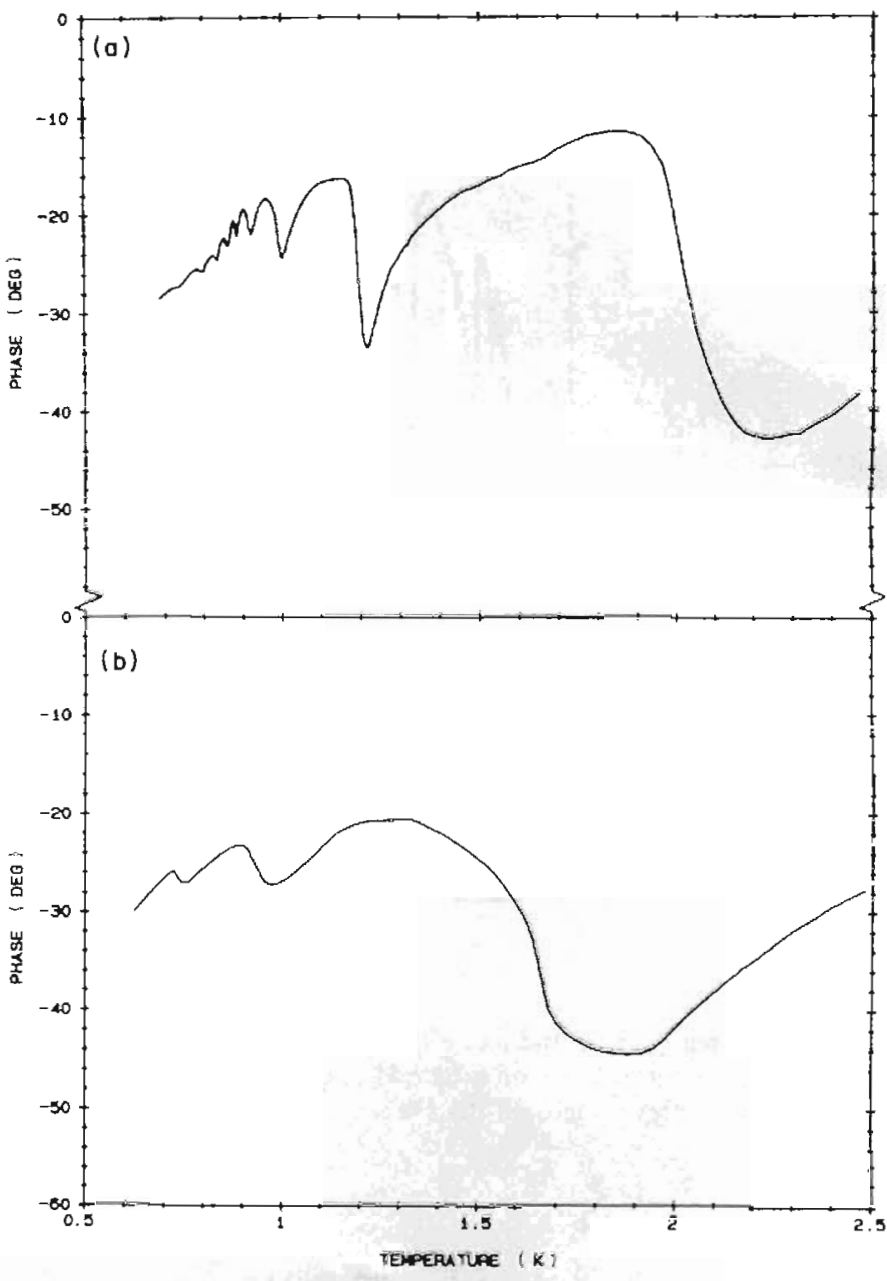

FIG. 6. (a) Part of the data for the ${ }^{4} \mathrm{He}$ film on $\mathrm{H}_{2}$ in Fig. 4, drawn on an expanded scale. (b) Similar run to (a), but with a reduced amount of ${ }^{4} \mathrm{He}$ in the cell $(p=0.115$ mbar at $5 \mathrm{~K}$ ).

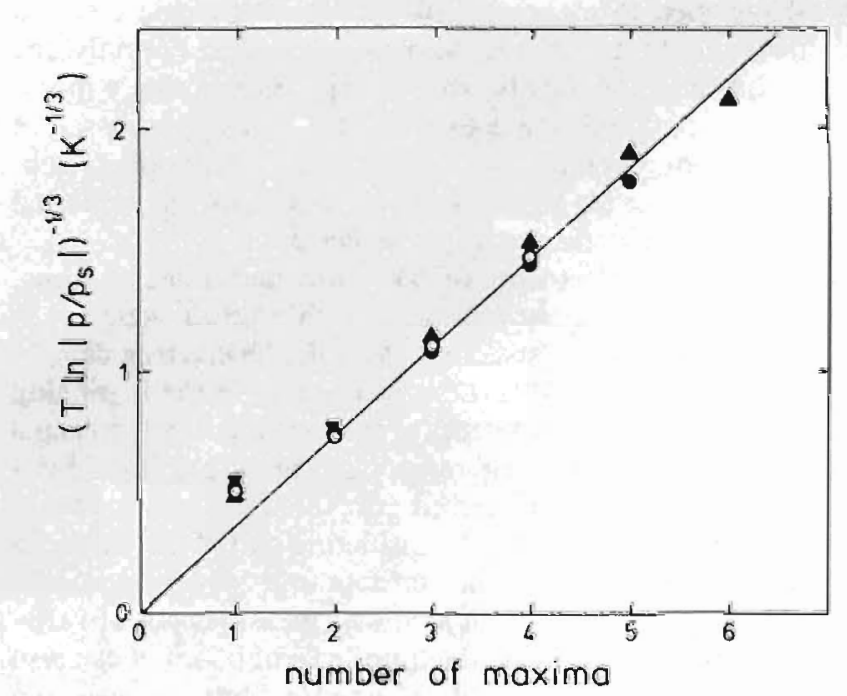

FIG. 7. Normalized van der Waals thickness $d /(\alpha)^{1 / 3}=(T$ In $\left.\left|p / p_{s}\right|\right)^{-1 / 3}$ plotted vs, the number of mobility maxima (which is equal to the number of monolayers according to the interpretation given here). The full symbols refer to runs at various arnounts of ${ }^{4} \mathrm{He}$, the open symbols are results of Paalanen and lye (ref. 12). The straight

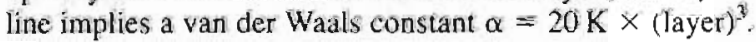




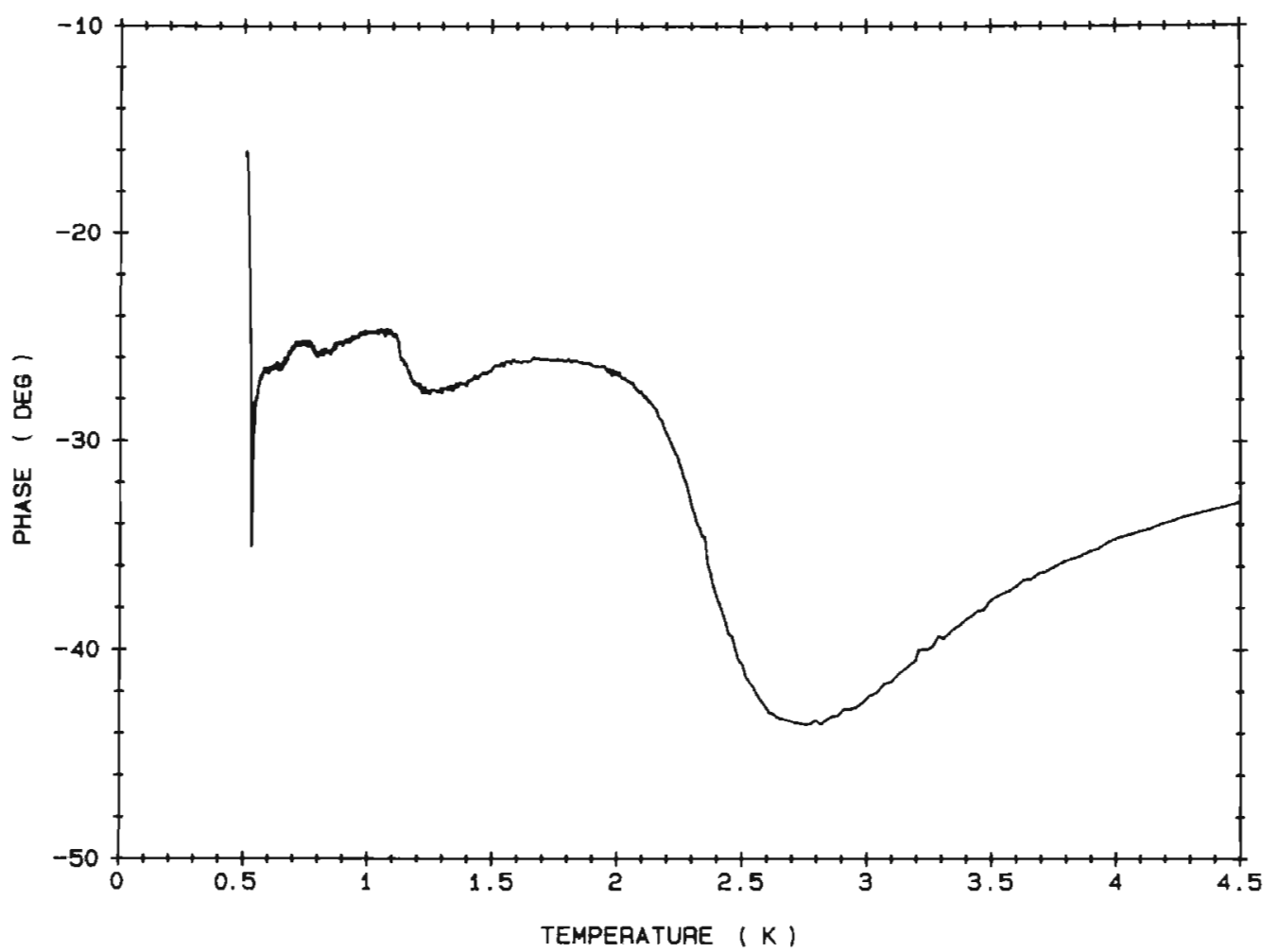

Fig. 8. Phase shift $\varphi$ for electrons above a ${ }^{3} \mathrm{He}$ film on solid $\mathrm{H}_{2}\left(p\left({ }^{3} \mathrm{He}\right)=3.5 \mathrm{mbar}\right.$ at $\left.5 \mathrm{~K}\right)$. As for ${ }^{4} \mathrm{He}$, the mobility maxima occur at those temperatures where completely filled monolayers are expected. The steep rise at $0.5 \mathrm{~K}$ signals the rapid increase of film thickness at that temperature.

opment of cracks resulting from thermal contraction of the hydrogen crystal, is unlikely because the anomalies occur both on cooling and on warming the sample. Further measurements are required to investigate this phenomenon.

\section{Solid $\mathrm{H}_{2}$ covered with thin He films}

The mobility of electrons on bare $\mathrm{H}_{2}$ was found to be limited to several times $10^{4} \mathrm{~cm}^{2} \mathrm{~V}^{-1} \mathrm{~s}^{-1}$ due to defect scattering. One might suppose that the defects can be "smoothed" and the mobility in this way improved by covering the $\mathrm{H}_{2}$ substrate with a thin $\mathrm{He}$ film. However, our experiments as well as earlier measurements by Paalanen and Iyc (henceforth referred to as PI) (12) show that on the contrary $\mu$ decreases when $\mathrm{He}$ is added. This behavior will now be discussed in some detail.

The effect is evident in Fig. 4 for temperatures below about $5 \mathrm{~K}$. In this run the sample cell contained a small constant amount of ${ }^{4} \mathrm{He}(0.37 \mathrm{mbar}$ at $5 \mathrm{~K})(1 \mathrm{bar}=100 \mathrm{kPa})$ in addition to the hydrogen. Whereas for $T>5 \mathrm{~K}$ all the helium was essentially in the gas phase ${ }^{2}$ (where its contribution to the scattering is negligible compared to that of the substrate, $\tau_{\mathrm{He}}^{-1} / \tau_{\mathrm{defect}}^{-1}<$ $10^{-2}$ ), at lower $T$ a film started to build, which at $T \sim 0.5 \mathrm{~K}$, where nearly all the helium had condensed, corresponded to 10 monolayers.

The growth of the film affects the mobility, but the mobility is not monotonic in the film thickness. Rather it displays pronounced oscillations that decrease in amplitude as $T$ is reduced and the film becomes thicker. Similar oscillations in $\mu$ were already observed by PI in an experiment where the ${ }^{4} \mathrm{He}$ film thickness was varied at constant $T$. These authors ascribed the mobility maxima to completed and the minima to half-filled $\mathrm{He}$

${ }^{2}$ For $T>5 \mathrm{~K}$ the data therefore represent a measurement on a bare substrate. monolayers, respectively. Four oscillations could be observed in their measurement at $1.45 \mathrm{~K}$, an indication that the layering of the He film extends over at least 4 monolayers. Our data show that at lower $T$ the layering extends even further, reaching over distances considerably larger than the short-range order known from bulk liquids (cf. Fig. 5a). This is illustrated in Fig. $6 a$ where data of Fig. 4 are plotted on an expanded scale. Nine oscillations can be resolved, which implies some kind of ordering in the film over 9 atomic distances.

That the oscillations in $\mu$ are indeed related to the film thickness and are not due to some other temperature dependence of the apparatus is demonstrated by the data in Fig. $6 b$, which were taken under the same conditions as in Fig. $6 a$ except for a reduced amount of ${ }^{4} \mathrm{He}$ in the cell $\left(P_{\mathrm{He}}=0.115\right.$ instead of 0.35 mbar at $5 \mathrm{~K}$ ). Consequently the maximum film thickness was limited to 3 layers, in good accord with the observed number of oscillations. As an additional support, we have plotted in Fig. 7 the data of several runs in a graph similar to one used by PI. The straight line represents the dependence expected for a "van der Waals film" whose thickness $d$ is related to the actual gas prcssure $p$ and the saturated vapor pressure $p_{\mathrm{s}}$ at the temperature $T$ by

[7] $\quad d^{3}=\alpha /\left(T \ln \left|p / p_{s}\right|\right)$

The van der Waals constant $\alpha$ for ${ }^{4} \mathrm{He}$ on solid $\mathrm{H}_{2}$ as determined from our results agrees perfectly with the value $\alpha=$

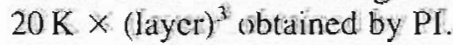

Although the correspondence between the mobility maxima and filled monolayers is thus clearly demonstrated, the origin of the reduction in $\mu$ for partly filled layers remains to be explained. Sources for the excess scattering could be the surface structure or excitations of the film. In the latter case the 


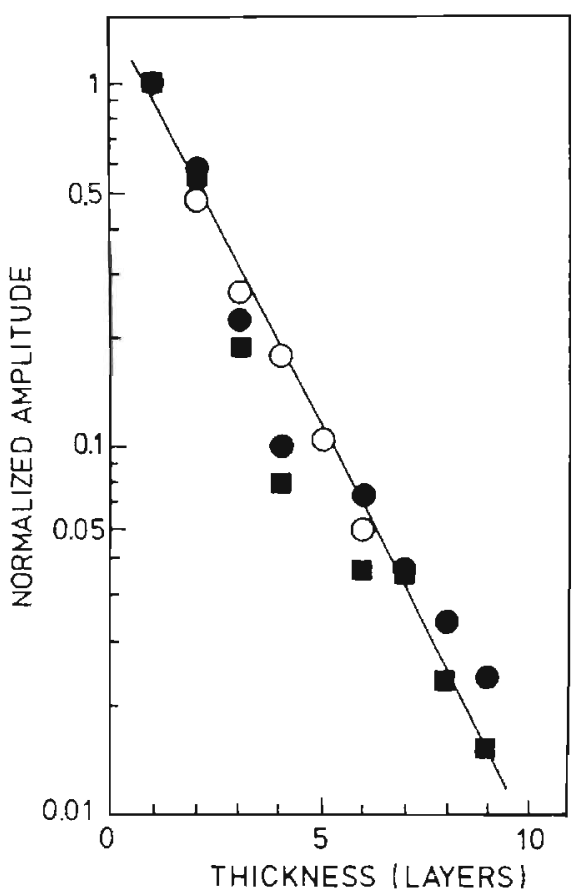

Fig. 9. Full symbols: amplitude of the modulation in the mobility as a function of the frim thickness, normalized to the value at the first layer. Open symbols: amplitude of the oscillations in the density distribution of a Lennard-Jones system as one moves from the solid into the liquid phase (from Broughton et al. (17)). The straight line represents an exponential decay of the amplitudes with a characteristic length of 2.5 layers

superfluidity of the ${ }^{4} \mathrm{He}$ films discussed so far might be relevant. For this reason we have also studied ${ }^{3} \mathrm{He}$ films, which in the temperature range of our measurement are not superfluid. We find that a modulation in the mobility is observed for ${ }^{3} \mathrm{He}$ as well (see Fig. 8); thus the oscillatory behavior of $\mu$ is not related to superfluidity.

An interpretation given by Pl for submonolayer films is that $\mu$ in this case is determined by the scattering from density fluctuations in the adsorbed 2D He system. This picture can be extended to thicker films, if one assumes that $\mathrm{He}$ forms welldefined layers as they are observed, for example, for krypton on graphite (13). A theory for the electron mobility in this transition region between the submonolayer range and very thick films, where scattering from ripplons dominates, does not yet exist. Therefore a quantitative description cannot be given at present. Nevertheless, our data allow us to draw some conclusions on the range of the correlation induced in the liquid film by the presence of the solid $\mathrm{H}_{2}$ wall. For this purpose we have plotted in Fig. 9 the amplitude of the modulation in $\mu$ vs. film thickness, which should yield information on the length scale of the layering. The data are compatible with a roughly exponential decay with a characteristic length (corresponding to a decrease by a factor of $e$ ) of about 2 atomic distances.

Indications for the layering of He films have been found also in specific heat and third sound measurements of ${ }^{4} \mathrm{He}$ on graphite substrates $(14,15)$. Up to 7 layers could be distinguished in these experiments. A theoretical study of He films on various substrates by Krotscheck (16) gave quite substantial layering as well. (Unfortunately these calculations are restricted to $n \leqslant 4$ layers and therefore cover only part of our experimental range.) More generally, computer simulations for Lennard-Jones sys- tems have shown that the solid-liquid interface of such systems is characterized by a transition region as much as 10 atomic distances wide (17). Over this length the density distribution, which is strongly peaked at the lattice positions in the solid, decays towards the constant value of the liquid (Fig. $5 b$ ). Thus the order induced by a plane interface extends much further into the liquid than one might have argued on the basis of the relatively short-range structure in the density correlation function for liquids in 3 dimensions.

For a comparison with our results we also plotted in Fig. 9 the amplitude of the density modulation near a van der Waals solid-liquid interface, taken from the density profile in Fig. $5 b$. The decay lengths for these two sets of data agree remarkably well; in view of the crudeness of the argument we do not want to overestimate this point, however. (Two aspects are to be taken into account in a more quantitative discussion: $(i)$ as the film thickness increases, the distance of the electrons from the surface grows and hence their coupling to the film decreases; (ii) correlation effects in the electronic motion can affect the mobility at the lower temperatures, which in Fig. 9 correspond to the larger values of $d$.)

\section{Conclusions}

The results presented above show that electrons on He films display a number of interesting features. A promising substrate for these films is solid $\mathrm{H}_{2}$, which interacts only weakly with the electrons and apparently can be grown with good surface quality. The films on this substrate show deviations from the bulk liquid, visible in pronounced mobility oscillations of surface electrons, which can be interpreted as layering in the film up to nearly 10 atomic distances. This, probably induced by the solid wall, is observed for both He isotopes. The nature of the layering with respect to structure and correlations in the film is not yet clear. Further experiments, such as precise measurements of the vapor pressure above the film, the propagation of third sound, and neutron and X-ray scattering, should yield complementary information. Besides, one might hope that the surface electrons, which have proven to be an extremely sensitive probe, will also add to the understanding of liquid films in future experiments.

\section{Acknowledgements}

We appreciate valuable contributions by W. Gombert, H. Etz, W. Idstein, and U. Albrecht. This work was begun when one of us (A.J.D.) was a Fulbright fellow on sabbatical leave in Mainz. He received partial support from NSF Grant No. DM3-82-13581. Financial support by the Deutsche Forschungsgemeinschaft is also gratefully acknowledged.

1. M. W. Cole. Rev. Mod. Phys. 46, 451 (1974); C. C. Grimes. Surf. Sci. 73, 379 (1978); V. S. EdelMan. Usp. Fiz. Nauk, 130, 675 (1980); Sov. Phys. Usp. (Engl. Transl.), 23, 227 (1980): F. I. B. Williams. Surf. Sci. 113, 371 (1982); Yu. P. MONARKha and V. B. Shikin. Fiz. Nizk. Temp. (Kiev), 8, 563 (1982); Sov. J. Low Temp. Phys. (Engl. Transl.), 8, 279 (1982).

2. R. Mehrotra, B. M. Guenin, and A. J. Dahm. Phys. Rev. Lett. 48, 641 (1982).

3. P. Leiderer. Physica $B \neq C$ (Amsterdam), 126, 92 (1984).

4. H. Etz, W. Gombert, W. Idstein, and P. Leiderer. Phys. Rev. Lett. 53, 2567 (1984)

5. W. T. Sommer. Ph.D. thesis, Stanford Univ. (1965); W. T. Sommer and D. J. Tannelr. Phys. Rev. Lett. 27, 1845 (1971).

6. S. A. Jackson and P. M. Platzman. Phys. Rev. B: Condens. Matter, 24, 499 (1981); O. Hipoltto, G. A. FarIas, and N. STUdART. Surf. Sci. 113, 394 (1982). 
7. E. Y. Andrei. Phys. Rev. Lett. 53, 1499 (1984).

8. A. M. Troyanovskil, A. P. Volodin, and M. S. Khaikin. Pis'ma Zh. Eksp. Teor. Fiz. 29, 421 (1979); JETP Lett. (Engl. Transl.). 29, 382 (1979); A. M. Troyanovskil and M. S. Khalkin. Zh. Eksp. Teor. Fiz. 81, 398 (1981); Sov. Phys. JETP (Engl. Transl.), 54, 214 (1981).

9. K. Kajita. J. Phys. Soc. Jpn. 52, 372 (1982); Surf. Sci. 142, 86 (1984)

10. V. S. Edel'man and M. I. Faby. J. Low Temp. Phys. 52, 301 (1983).

11. J. WEEKS. In Strongly fluctuating condensed systems. Edited by T. Riste. (Plenum, 1980); D. Nenow. Prog. Cryst. Growth Charact. 9.185 (1984).
12. M. A. PaAlanen and Y. Iye. Phys. Rev. Lett. 55, 1761 (1985).

13. A. Thomy, X. Duval, and J. Regnier. Surf. Sci. Rep. 1, 1 (1981).

14. S. E. Polanco, J. H. Quateman, and M. Bretz. J. Phys. (Colloq.), 6, 344 (1978).

15. J. D. Maynard and M. H. W. Chan. Physica B + C (Amsterdam), 109 and 110, 2090 (1982).

16. E. Krotscheck. Phys. Rev. B: Condens. Matter, 32, 5713 (1985).

17. J. Q. Broughton, A. Bonissent, and F. F. Abraham. J. Chem. Phys. 74, 4029 (1981). 